\title{
Scintigraphic Assessment of Radio-Aerosol Pulmonary Deposition With the Acapella Positive Expiratory Pressure Device and Various Nebulizer Configurations
}

\author{
Fabrício O S Mesquita MSc, Valdecir C Galindo-Filho PhD, João Luis F Neto PT, \\ André M Galvão MSc, Simone C S Brandão MD PhD, \\ James B Fink PhD FAARC, and Armèle Dornelas-de-Andrade PhD
}

\begin{abstract}
BACKGROUND: The Acapella device produces high-frequency oscillations and positive expiratory pressure to promote bronchial secretion clearance. Its performance during aerosol delivery has not been described. We evaluated the effect of nebulizer and Acapella configuration on pulmonary deposition of radio-tagged aerosol in healthy subjects. METHODS: Ten healthy male subjects (mean age $24.4 \pm 2.2 \mathrm{y}$ ) participated in a crossover study that compared pulmonary delivery of $4 \mathrm{~mL}$ of technetium-99m-labeled diethylene triamine penta-acetic acid $(25 \mathrm{mCi})$ and $0.9 \%$ saline solution via jet nebulizer. We tested 3 configurations: nebulizer attached to the distal end of the Acapella; nebulizer placed between the mouthpiece and the Acapella; and nebulizer alone (control). With scintigraphy we measured radio-aerosol deposition in 6 lung regions: upper, middle, lower, central, intermediate, and peripheral. RESULTS: Deposition was similar between the right and left lungs, with no significant differences between device configurations. Lung deposition was less with the nebulizer attached to the Acapella than with nebulizer between the mouthpiece and the Acapella $(P=.001$, for both lungs) or without the Acapella $(P=.003$ and $P=.001$ for the right and left lungs, respectively). There was no significant difference between the setup without Acapella and the setup with the nebulizer between the mouthpiece and the Acapella $(P=.001$, for both lungs $)$. On the vertical axis, deposition was lower with the nebulizer attached to the distal end of the Acapella than with the nebulizer between the mouthpiece and the Acapella (upper region $P<.001$, middle region $P=.001$, lower region $P=.003)$, and lower with the nebulizer attached to the distal end of the Acapella than with the setup without Acapella (upper and middle region both $P=.001$, lower region $P=.002$ ), with up to a 3-fold difference in the middle and lower regions. On the centralperipheral axis, deposition was lower with the nebulizer attached to the distal end of the Acapella than with the nebulizer between the mouthpiece and the Acapella (central region $P<.001$, peripheral region $P<.001$ ), and lower with the nebulizer attached to the distal end of the Acapella than with the setup without Acapella (central and peripheral regions both $P=.002$ ), with differences of 3-4-fold between the central and peripheral regions. CONCLUSIONS: Placing the nebulizer distal to the Acapella, as recommended by the manufacturer, decreased intrapulmonary deposition, compared to placing the nebulizer between the Acapella and the patient airway, or delivering aerosol without the Acapella in the circuit. (ClinicalTrials.gov NCT01102166) Key words: nebulizer; aerosol; pulmonary scintigraphy; aerosol deposition; positive expiratory pressure; oscillatory PEP. [Respir Care 2014;59(3):328-333. (C) 2014 Daedalus Enterprises]
\end{abstract}

Mr Mesquita, Dr Galindo-Filho, Mr Neto, Mr Galvão, Dr Brandão, and Dr Dornelas-de-Andrade are affiliated with the Department of Physical Therapy, and Dr Brandão who is affiliated with the Nuclear Department,
Universidade Federal de Pernambuco, Recife City, Brazil. Dr Fink is affiliated with the Division of Respiratory Therapy, School of Health Professions, Georgia State University, Atlanta, Georgia and is an independent consultant in San Mateo, California. 


\section{Introduction}

The clearance of pulmonary secretions is directly affected by changes in cross-sectional area of the airways, the composition and production of mucus in the respiratory tract, ciliary function, and cough reflex. ${ }^{1}$ Inflammatory and infectious diseases or dyskinetic syndromes such as asthma, chronic bronchitis, and cystic fibrosis can involve hypersecretion and blockage of the airways by mucus. ${ }^{2}$ The retention of lung secretions can result in complete or partial obstruction of the airways, which results in atelectasis, lung hyperinflation, and increased respiratory muscle load. ${ }^{3}$

\section{See the Related Editorial on Page 441}

Inhalation of medical aerosols promotes hydration and reduction of secretions viscosity, optimizing mucociliary clearance. ${ }^{4}$ Thus, inhalation of drugs transported as inhalable particles may alter mucus rheology and has become clinically accepted as an adjunct to physiotherapy in the treatment of chronic lung conditions. ${ }^{5}$ In addition, inhalation increases mucociliary clearance by influencing ciliary function, increasing osmotic drive, and altering mucus viscoelasticity. This results in reduction of viscosity and augmentation of sputum clearance. ${ }^{6}$

Bronchial hygiene therapy involves the use of noninvasive and invasive techniques to assist the mobilization and clearance of secretions. ${ }^{7}$ The methods of bronchial hygiene reported in the literature include positive expiratory pressure (PEP) and high-frequency oscillation of the airway. The principle of PEP is based on generating positive pressure to splint open and stabilize airways, preventing bronchial collapse. PEP is also thought to improve collateral ventilation to allow better distribution of gas beyond occluded airways. ${ }^{7}$ Oscillatory PEP may have added benefit over the PEP mask alone in helping to dislodge thick secretions from the airway walls

This study was partly supported by grants from Fundação de Amparo à Ciência e a Pesquisa and Conselho Nacional de Desenvolvimento Científico e Tecnológico.

Valdecir C Galindo-Filho $\mathrm{PhD}$ presented a version of this paper at the annual meeting of the European Respiratory Society, held September 1519, 2007, in Stockholm, Sweden.

Dr Fink is a consultant to Aerogen, Bayer, Boehringer Ingelheim, Cubist, Dance Biopharm, Novartis, ONY, Parion, Aridis, and the WHO.

Correspondence: Armèle Dornelas-de-Andrade PhD, Department of Physical Therapy, Universidade Federal de Pernambuco, Avenida Jornalista Aníbal Fernandes, Recife, Pernambuco, Brazil, 50670-901. E-mail: armeledornelas@yahoo.com.

DOI: $10.4187 /$ respcare.02291

\section{QUICK LOOK}

\section{Current knowledge}

Positive expiratory pressure (PEP) and oscillatory PEP devices are commonly used to aid bronchial secretion clearance. Delivering aerosol therapy during PEP may alter the aerosol and the aerosol delivery.

\section{What this paper contributes to our knowledge}

Placing the jet nebulizer distal to the PEP device decreased pulmonary deposition, compared to placing the nebulizer between the patient and the PEP device, or using the nebulizer alone.

and decrease mucus viscoelasticity. ${ }^{8,9}$ High-frequency oscillation of both the chest wall and airway have been shown to facilitate secretion clearance. ${ }^{10}$

The Acapella high-frequency oscillatory PEP device (Smiths Medical, Watford, United Kingdom) produces oscillations during exhalation by intermittently occluding the air flow with a magnetic, adjustable valve that allows adjustment of frequency, oscillation amplitude, and mean pressure through 5 levels, via a dial on the device. It is suggested that expectoration can be optimized when the applied pressure frequency coincides with the ciliary movement, approximately $13 \mathrm{~Hz} .{ }^{10}$ Acapella can be used in any position, does not depend on the slope or level of PEP, and allows concomitant aerosol administration. ${ }^{11}$

We hypothesized that the placement of the nebulizer distal to the Acapella, as recommended by the manufacturer, would reduce aerosol lung delivery, compared to more proximal placement (between device and mouthpiece) or compared to nebulizer alone. The aim of this study was to evaluate the effect of aerosol generator placement in 2 different positions during use of the Acapella device on delivery of radio-labeled aerosol to the lungs of healthy subjects. This is the first study to analyze radio-aerosol deposition in normal subjects with and without the Acapella.

\section{Methods}

This randomized crossover clinical trial received approval from the institutional ethics and human research committee at the Universidade Federal de Pernambuco, and subjects gave written informed consent. The study was performed at Português Hospital, Recife City, Brazil.

\section{Subjects}

The inclusion criteria were: no history of asthma or other lung disease; age 18-30 years; and $\mathrm{FVC}, \mathrm{FEV}_{1}$, and 


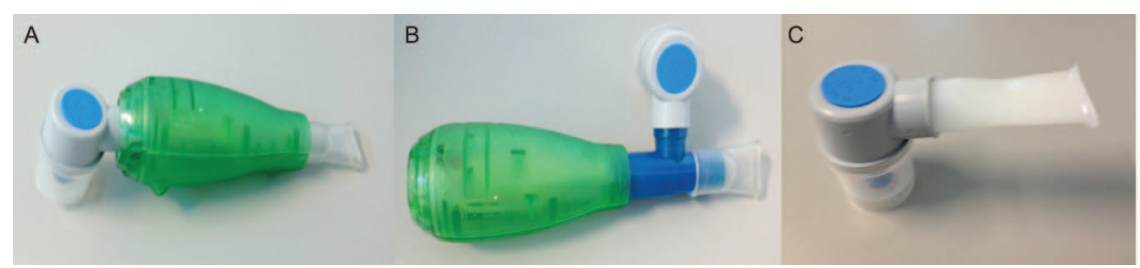

Fig. 1. Test configurations. A: Nebulizer attached to the distal end of the Acapella. B: Nebulizer attached via T-piece to the proximal end of the Acapella. C: Control setup: nebulizer and mouthpiece without Acapella.

peak expiratory flow all $\geq 80 \%$ of predicted. The exclusion criteria were history of smoking and signs or symptoms of illness (fever, tachycardia, and tachypnea) or respiratory disease.

\section{Procedures}

A jet nebulizer (ST3, NS, São Paulo, Brazil) operated at $7 \mathrm{~L} / \mathrm{min}$ with compressed air was used in all configurations. The nebulizer's mass median aerodynamic diameter (MMAD, $3.6 \mu \mathrm{m})$ and geometric standard deviation of $(2.2 \mu \mathrm{m})$ were previously determined with via cascade impactor, using standard United States Pharmacopoeia methods.

\section{Device Configurations}

The subjects were randomly allocated to receive aerosol with 3 device configurations (Fig 1):

A: With the nebulizer attached to the distal end of the Acapella, per the manufacturer's recommendation (distal)

B: With the nebulizer connected to a T-piece between the mouthpiece and the Acapella (proximal)

C: With the nebulizer attached to a standard mouthpiece, without the Acapella in the circuit (control)

A clinical assessment was performed and data measurements included age, weight, height, body mass index, spirometry, and maximal inspiratory pressure. FVC, $\mathrm{FEV}_{1}$, and peak expiratory flow were measured with a spirometer (2110, Vitalograph, Buckinghamshire, United Kingdom) according to the American Thoracic Society standards. ${ }^{12}$ Maximal inspiratory pressure was measured with a manometer (MV-150, Ger-Ar Comércio de Produtos Médicos, São Paulo, Brazil), with the subject seated and with a nose clip, and instructed to perform a maximal inspiration during a minimum of 1 second. ${ }^{13}$ Three measurements were performed and the highest value was retained for analysis.

The scintigraphy procedures were developed in the Department of Nuclear Medicine. The subjects performed aerosol inhalation based on the randomization schedule, and then underwent scintigraphy. Four milliliters of $0.9 \%$ physiologic saline solution containing technetium-99mlabeled diethylene triamine penta-acetic acid $(25 \mathrm{mCi})$ was nebulized over $9 \mathrm{~min}$. The aerosol was inhaled via mouth-
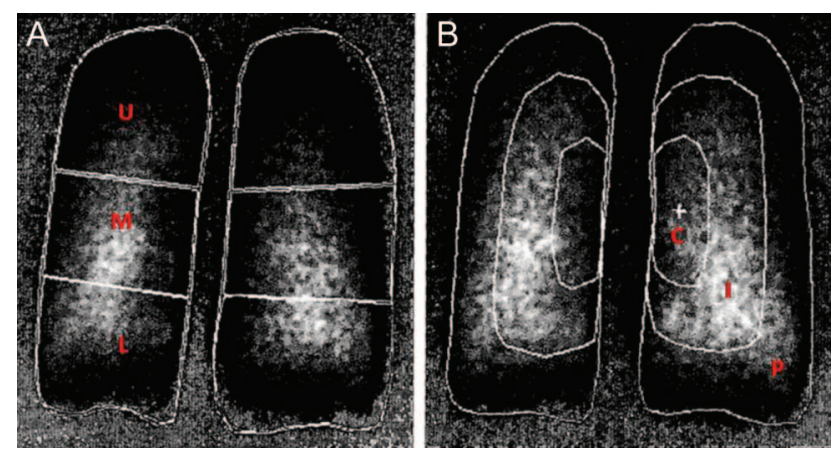

Fig. 2. Lung regions of interest. A: Upper third (U), middle third (M), and lower third (L). B: Central region (C), intermediate (I), and peripheral region $(\mathrm{P})$.

piece, with the subject seated upright. All the subjects were previously trained for deep breathing and inspiratory pause. ${ }^{14}$

Immediately after aerosol administration scintigraphy was performed with a gamma camera (ADAC Forte, Philips, Eindhoven, The Netherlands), during a period of 300 seconds, using a matrix of $256 \times 256 \times 16$ in the posterior position.

The lung images were divided into regions of interest vertically (upper, middle, and lower thirds) and on the centralperipheral axis (central, intermediate, and peripheral) (Fig. 2), and the radiation counts in the regions of interest were calculated. ${ }^{15}$ With each subject a wash-out period minimum of 7 days was used between radio-labeled aerosol exposure, to ensure clearance of the inhaled radioactive material.

\section{Statistical Analysis}

For statistical analysis the Kolmogorov-Smirnov test was used to analyze normality, followed by analysis of variance and the Tukey post hoc test, in statistics software (SPSS 18.0, SPSS, Chicago, Illinois). The results are expressed as mean $\pm \mathrm{SD}$, and we considered differences statistically significant at $P<.05$.

\section{Results}

Of the 14 healthy males we included, only 10 completed the protocol: 2 dropped out due to claustrophobia, and 2 retracted consent. The mean values in the 10 subjects who completed the protocol were: age $24.4 \pm 2.2$ years, body mass index $22.6 \pm 2.6 \mathrm{~kg} / \mathrm{m}^{2}$, peak expiratory flow 


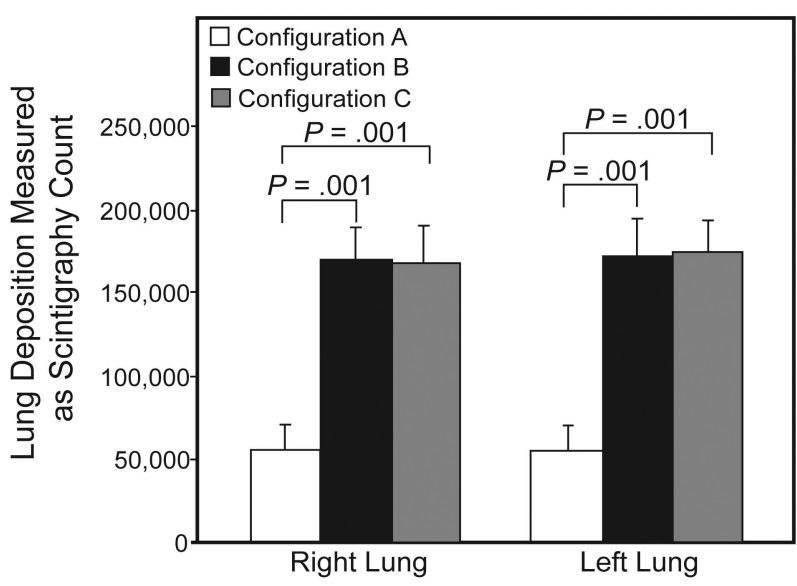

Fig. 3. Radio-aerosol pulmonary deposition in the right versus left lungs. ${ }^{*} P=.001$ via analysis of variance and Tukey post hoc test. The tops of the data bars represent the means. The whisker bars represent the standard deviations.

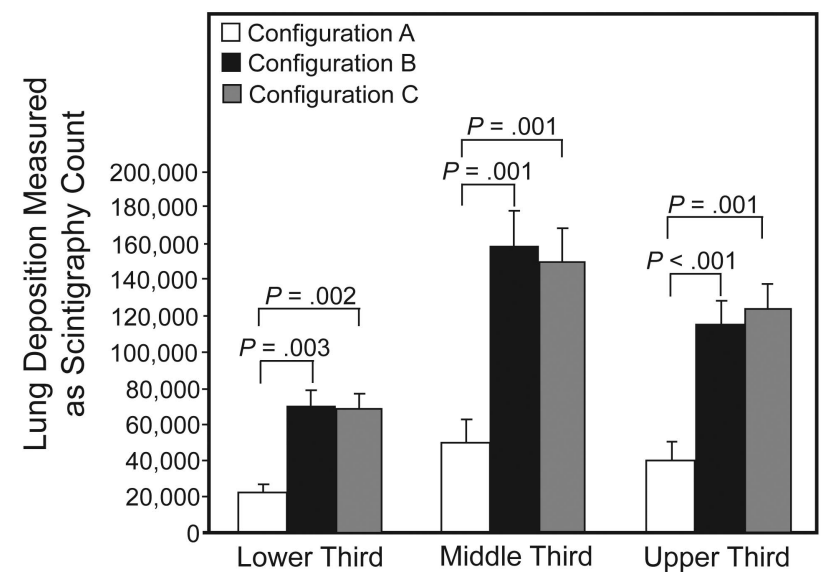

Fig. 4. Radio-aerosol pulmonary deposition in the upper, middle, and lower thirds of the right lung. The tops of the data bars represent the means. The whisker bars represent the standard deviations.

$583 \pm 41 \mathrm{~L} / \mathrm{min}, \mathrm{FEV}_{1} 4.3 \pm 0.5 \mathrm{~L}, \mathrm{FVC} 4.7 \pm 0.6 \mathrm{~L}$, and maximal inspiratory pressure $110 \pm 16 \mathrm{~cm} \mathrm{H}_{2} \mathrm{O}$.

Deposition in the right and left lungs was similar with all the configurations studied. There was less total deposition with configuration A than with $\mathrm{B}$ or $\mathrm{C}$ (Fig. 3). There was no significant difference between configuration $\mathrm{B}$ and $\mathrm{C}$. The differences between the regions of interest were up 3-fold greater with B and C, compared to A.

All 3 vertical regions (upper, middle, and lower third) had less aerosol deposition, in both lungs, with A than with B and C (Fig. 4). The ratio of upper to lower deposition was marginally higher with $\mathrm{A}(0.70 \pm 0.21)$ than with $\mathrm{B}$ or $\mathrm{C}(0.6 \pm 0.11$ and $0.58 \pm 0.12$, respectively). Similarly, the central-peripheral axis regions showed less deposition with configuration A than with B or C (Fig. 5). The ratio of central to peripheral deposition trended lower

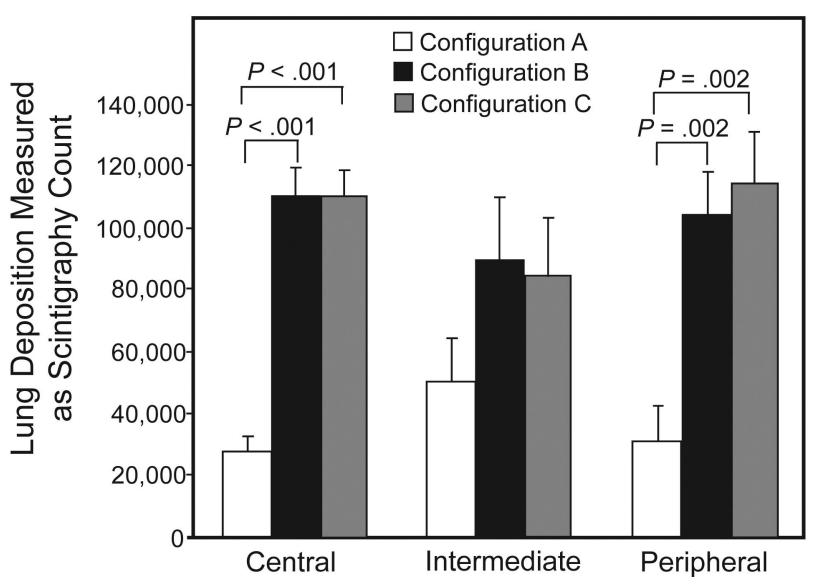

Fig. 5. Radio-aerosol pulmonary deposition in the central, intermediate, and peripheral regions of the right lung. The tops of the data bars represent the means. The whisker bars represent the standard deviations.

with configuration $\mathrm{A}(0.89 \pm 0.11)$ than with $\mathrm{B}$ or $\mathrm{C}$ $(1.05 \pm 0.23$ and $0.93 \pm 0.12$, respectively).

The distribution of radio-aerosol deposition inside the Acapella differed with the nebulizer at the inlet of the Acapella versus at the outlet (Fig. 6).

\section{Discussion}

Placing the nebulizer at the distal end of the Acapella significantly decreased pulmonary deposition of radioaerosol, compared to placing the nebulizer at the proximal end of the Acapella, and compared to nebulizer alone, in both the right and left lungs. The primary reason for the lower pulmonary deposition of radio-aerosol with distal nebulizer placement is likely the inertial impaction of larger aerosol particles as they pass through the Acapella, which makes less aerosol available for inhalation. Internal mechanisms that create turbulent or transitional flow increase the deposition of larger particles. ${ }^{10}$ This is supported by the report of Berlinski and Hayden, ${ }^{16}$ who evaluated changes in aerosol particle size from 2 continuously operated jet nebulizers with several PEP and oscillatory PEP devices. The MMAD of aerosol produced by the Hudson nebulizer $(4.1 \mu \mathrm{m})$ was reduced to $1.2 \mu \mathrm{m}$ with both the Acapella Choice and Acapella Duet, when used as recommended by the manufacturer (distal and inferior, respectively). This supports our supposition that the internal mechanisms of an oscillatory PEP device filter larger aerosol particles, reducing the total dose of aerosol available for inhalation (however, this was not reported). This reduction in particle size was associated with an increase of fineparticle fraction $(<5 \mu \mathrm{m})$, from $59 \%$ to $>87 \%$, which might be expected to have a greater peripheral deposition than the aerosol with the larger MMAD produced by the 

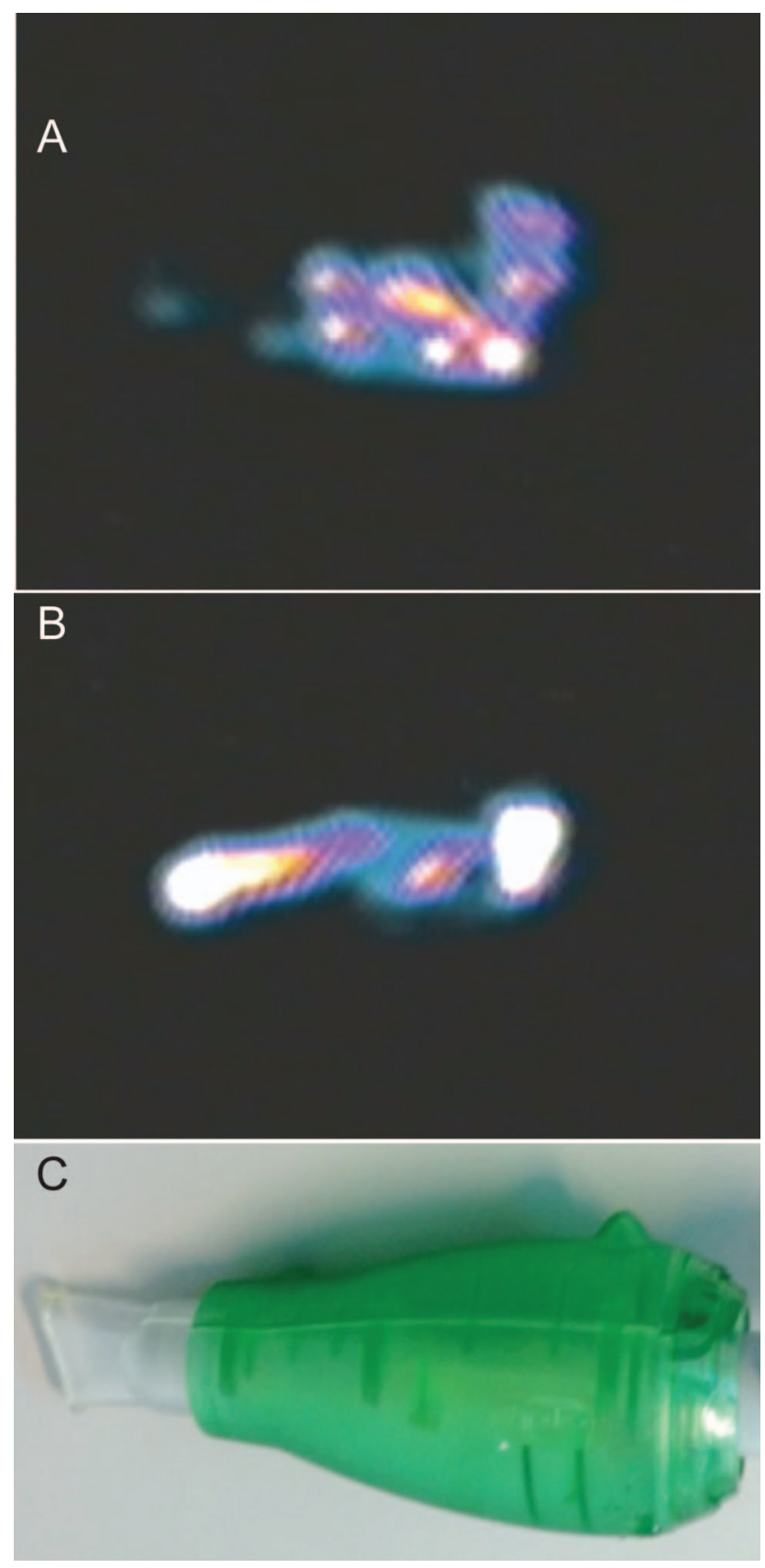

Fig. 6. Radio-aerosol distribution inside the Acapella with $(A)$ the nebulizer attached to the distal end of the Acapella, (B) the nebulizer attached to the proximal end of the Acapella. C: Orientation of the Acapella in Figures 6A and 6B.

nebulizer alone. This is consistently greater peripheral deposition, or a lower central-to-peripheral deposition ratio. In testing of the Pari LC Plus nebulizer with its proprietary PEP valve at settings of 1.5 to 4.5 , Berlinski and Hayden ${ }^{16}$ found no changes in MMAD or geometric standard deviation. The Pari PEP systems did not change the nebulized albuterol characteristics.
We did not find the hypothesized difference between proximal nebulizer placement (between the Acapella device and the patient) and the nebulizer alone in the normal subjects tested. This suggests that placing the nebulizer between the Acapella and the mouthpiece did not substantially change the fraction of deposited dose in the lung, versus control.

Laube et $\mathrm{al}^{17}$ compared pulmonary delivery of radiolabeled aerosol delivery from a jet nebulizer (Pari LC Plus) with and without PEP in patients with cystic fibrosis. They reported reduced pulmonary delivery with PEP, and a modestly lower central/peripheral ratio, compared to nebulizer alone. Our findings are consistent to the point that we also had a modest trend toward lower central/peripheral ratio and reduced pulmonary deposition with Acapella in configuration $\mathrm{A}$, but not with $\mathrm{B}$ or $\mathrm{C}$.

In contrast to Berlinski and Hayden, ${ }^{16}$ Laube et al ${ }^{17}$ reported a reduced MMAD of $3.3 \mu \mathrm{m}$ with PEP and $4.07 \mu \mathrm{m}$ without PEP, with similar geometric standard deviations (2.61 $\mu \mathrm{m}$ and $2.78 \mu \mathrm{m}$, respectively). This lower MMAD may account for the difference in both pulmonary deposition and distribution. Those authors used a controlled breathing pattern during PEP exhalation, to maintain a target during exhalation, which may have increased the expiratory time and reduced the percent of aerosol inhaled from continuous nebulization, resulting in the reduction versus nebulizer alone. They did not find the expected distribution difference to the periphery or upper regions of interest in their population of patients with severe cystic fibrosis lung disease.

In normal subjects we also did not find the expected level of increase in peripheral or upper-lobe distribution with Acapella, in either configuration. This might be due to the nature of the normal condition of the airways, so Acapella did not have "floppy" airways to splint or obstructed airways for collateral ventilation to impact distal distribution.

We believe that the lower deposition of radio-aerosol found with the manufacturer-recommended distal position can be attributed to particle impaction inside the Acapella, ${ }^{11}$ which has several small-diameter internal ducts that increase the likelihood of impaction.

We found higher deposition in the middle and lower thirds than in the upper third, with all 3 configurations. This distribution in normal subjects may be attributed mainly to the vertical difference in pleural pressure between the upper and lower lung regions. ${ }^{18}$

According to Alderson and Line, ${ }^{19}$ other factors also influence the distribution of particles into the lungs, such as minute ventilation, inspiratory pressure, and nebulization position during inhalation. Ventilation per unit volume decreases from the base to the apex of the lung, as a result of regional differences in intrapleural pressure resulting from gravitational influences. ${ }^{18}$

In the lung, the gravitational and retractable forces act in the same direction, making the subatmospheric intrapleural pressure more negative in the apex than in the lung 


\section{Scintigraphic Assessment of Radio-Aerosol Pulmonary Deposition}

base, where the gravitational and retractable forces act in opposite directions. As a result, alveoli in the apex become more expanded and less compliant, with a small change in volume during inspiration. Moreover, alveoli in the base are less expanded and more compliant and have a greater change in volume during inspiration. ${ }^{18}$ In addition, our findings corroborate a study of radio-aerosol deposition coupled with bi-level ventilation in normal subjects. ${ }^{14}$

We found more deposition in the intermediate and peripheral regions than in the central region with all 3 configurations. The flow favors deposition in the large airways, owing to the inertial impact and possibly the greater radio-aerosol deposition in those areas is related to the aerosol particle size. ${ }^{19-22}$ Another important consideration is that the intrapleural pressure change is relatively uniform on the central-peripheral axis, so there is no centralperipheral change in ventilation per unit alveolar volume in the upright position. ${ }^{18}$

We used normal subjects to have a homogenous population in which to determine the impact of device configuration on pulmonary deposition, without having to account for differences in disease severity. It was beyond the scope of this study to isolate variables in obstructive pulmonary diseases that might impact aerosol distribution during oscillatory PEP. As our objective was to determine differences in distribution in the lungs and across regions of interest, we did not perform a mass balance to express lung deposition as a percentage of nominal dose.

\section{Conclusions}

Placing the nebulizer distal to the oscillatory PEP device decreased intrapulmonary deposition, compared to proximal placement, and compared to nebulizer alone.

\section{ACKNOWLEDGMENTS}

We are grateful to Maria José Clemente Menezes, Real Imagem, Hospital Português, for support during data collection.

\section{REFERENCES}

1. Van der Schans CP. Bronchial mucus transport. Respir Care 2007; 52(9):1150-1156.

2. Ragavan AJ, Evrensel CA, Krumpe P. Interactions of airflow oscillation, tracheal inclination and mucus elasticity significantly improve simulated cough clearance, Chest 2010;137(2):355-361.

3. Bilton D. Up date on non cystic fibrosis bronchiectasis. Curr Opin Pulm Med 2008;14(6):595-599.

4. Fink JB. Humidity and aerosol therapy In: Cairo JM, Pilbeam SP. Mosby's Respiratory Care Equipment, 8th edition. St Louis: Mosby; 2009:88-143.
5. Kellett F, Redfern J, Niven RM. Evaluation of nebulised hypertonic saline (7\%) as an adjunct to physiotherapy in patients with stable bronchiectasis. Respir Med 2005;99(1):27-31.

6. Hardy KA, Anderson BD. Noninvasive clearance of airway secretions. Respir Care Clin N Am 1996;2(2):323-345.

7. Rogers D, Doull IJ. Physiological principles of airway clearance techniques used in the physiotherapy management of cystic fibrosis. Curr Paediatr 2005;15(3):223-238.

8. Andersen JB, Qvist J, Kann T. Recruiting collapsed lung through collateral channels with positive end-expiratory pressure. Scand J Respir Dis 1979;60(5):260-266.

9. Darbee JC, Ohtake PJ, Grant BJB, Cerny FJ. Physiologic evidence for the efficacy of positive expiratory pressure as an airway clearance technique in patients with cystic fibrosis. Phys Ther 2004;84(6):524-537.

10. Alves Silva CE, Santos JG, Jansen JM, Melo PL. Laboratory evaluation of the Acapella device: pressure characteristics under different conditions, and a software tool to optimize its practical use. Respir Care 2009;54(11):1480-1487.

11. Volsko TA, Difiore JM, Chatburn RL. Performance comparison of two oscillating positive expiratory pressure devices: Acapella versus Flutter. Respir Care 2003;48(2):124-130.

12. American Thoracic Society statement. Snowbird workshop on standardization of spirometry. Am Rev Respir Dis 1979;119(5):831-838.

13. American Thoracic Society/European Respiratory Society. Statement on respiratory muscle testing. Am J Respir Crit Care Med 2002; 166(4):518-624.

14. França EE, Dornelas de Andrade AF, Cabral G, Almeida Filho P, Silva KC, Galindo Filho VC, et al. Nebulization associated with bi-level noninvasive ventilation: analysis of pulmonary radioaerosol deposition. Respir Med 2006;100(4):721-728.

15. Nobre MEP, Lopes F, Cordeiro L, Marinho PE, Silva TN, Amorim $\mathrm{C}$, et al. Inspiratory muscle endurance testing: pulmonary ventilation and electromyographic analysis. Respir Physiol Neurobiol 2007; 155(1):41-48.

16. Berlinski A, Hayden JB. Effect of concomitant use of positive expiratory pressure devices and nebulizers on nebulized albuterol characteristics. Ped Pulmonology 2010;(Suppl 33):379.

17. Laube BL, Geller DE, Lin TC, Dalby RN, Diener-West M, Zeitlin PL. Positive expiratory pressure changes aerosol distribution in patients with cystic fibrosis. Respir Care 2005 Nov;50(11):1438-1444.

18. Hayes M, Taplin GV. Lung imaging with radioaerossols for the assessment of airway disease. Semin Nucl Med 1980;10(3):243-250.

19. Alderson PO, Line BR. Scintigraphic evaluation of regional pulmonary ventilation. Semin Nucl Med 1980;10(3):218-240.

20. Fauroux B, Boulé M, Lofaso F, Zérah F, Clément A, Harf A, et al. Chest physiotherapy in cystic fibrosis: improved tolerance with nasal pressure support ventilation. Pediatrics 1999;103(3):e32.

21. Heyder J, Gebbart J, Rudolf G, Stahlhofen W. Physical factors determining particle deposition in the human respiratory tract. J Aerosol Sci 1980;11(5-6):505-15.

22. Fauroux B, Itti E, Pegeot J, Isabey, Meignan M, Ferry G, et al. Optimization of aerosol deposition by pressure support in children with cystic fibrosis: an experimental and clinical study. Am J Respir Crit Care Med 2000;162(6):2265-2271.

This article is approved for Continuing Respiratory Care Education credit. For information and to obtain your CRCE

(free to AARC members) visit

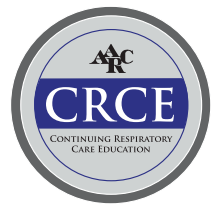

www.rcjournal.com 